\title{
Angular Fulde-Ferrell-Larkin-Ovchinnikov superfluid due to self-one-dimensionalization in imbalanced cold fermion gases
}

\author{
Youichi Yanase \\ Department of Physics, University of Tokyo, Tokyo 113-0033, Japan \\ and Department of Physics, Niigata University, Niigata 950-2181, Japan
}

\section{Abstract}

We study the angular Fulde-Ferrell-Larkin-Ovchinnikov (FFLO) state, in which the rotation symmetry is spontaneously broken, in population imbalanced fermion gases. The superfluid gases at near $T=0$ are investigated on the basis of the Bogoliubov-de Gennes (BdG) equation. We find that the angular FFLO state is stabilized in the gases confined in the toroidal trap, but not in the harmonic trap. We discuss the mechanism of the angular FFLO state based on the self-one-dimensionalization of the superfluid gas.

Key words:

PACS: 71.10.Ca, 03.75.Hh, 03.75.Ss, 05.30.Fk

\section{Introduction}

Cold fermion gases provide vast opportunities to study novel quantum condensed states [1]. One of the goals of current studies is the realization of the FFLO state [2, 3] in population imbalanced superfluid gases [4, 5]. However, no experimental evidence has been obtained for the FFLO state in cold fermion gases. This is mainly because the cold atom gases lack the translation symmetry owing to the trap potential, and therefore the spontaneous breaking of the space symmetry has never been observed. Then, it is difficult to differentiate the FFLO state from the phase separation [6].

Although the translation symmetry is absent in cold fermion gases, the rotation symmetry can be present. Therefore, it is highly desired to produce and study the FFLO state with broken rotation symmetry. For this purpose, we have studied the angular FFLO (A-FFLO) state in which the rotation symmetry is spontaneously broken [7, 8]. We proposed that the A-FFLO state is realized in the toroidal trap, although the harmonic trap cannot produce the A-FFLO state. In other words, the FFLO state has not been observed in cold fermion gases, because the experiments were carried out by using the harmonic trap. Therefore, it is highly desired to investigate the superfluidity in the toroidal trap. In this paper, we clarify the mechanism of the A-FFLO state in the toroidal trap, and discuss why the A-FFLO state is not realized in the harmonic trap.

\section{Model and Results}

To discuss the imbalanced fermion gases in the trap, we adopt the two-dimensional lattice Hamiltonian given as

$$
H=-t \sum_{<\vec{r}, \vec{r}^{\prime}>, \sigma} c_{\vec{r}, \sigma}^{\dagger} c_{\overrightarrow{r^{\prime}}, \sigma}+\sum_{\vec{r} \sigma}\left(V\left(\left|\vec{r}-\vec{r}_{0}\right|\right)-\mu_{\sigma}\right) n_{\vec{r}, \sigma}
$$

Preprint submitted to Physica C

$$
+U \sum_{\vec{r}} n_{\vec{r}, 1} n_{\vec{r}, 2}
$$

where $\sigma=1,2$ denote two hyperfine states, $\vec{r}_{0}$ is the center of the trap, and $n_{\vec{r}, \sigma}=c_{\vec{r}, \sigma}^{\dagger} c_{\vec{r}, \sigma}$ is the number operator of $\sigma$ particles. The two-dimensional gas is produced by the onedimensional optical lattice along the axial direction as well as by the pancake potential $\omega_{\mathrm{z}} \gg \omega_{\perp}$ with $\omega_{\mathrm{z}}$ and $\omega_{\perp}$ being the harmonic trap frequency along the axial and radial direction, respectively. We take the unit $\hbar=c=1$. The symbol $\left\langle\vec{r}, \overrightarrow{r^{\prime}}\right\rangle$ denotes the summation over nearest neighbour sites. The chemical potential $\mu_{\sigma}$ for $\sigma$ particles is determined so that the particle number of each state is $N_{\sigma}$. The particle number and the imbalance are expressed as $N=N_{1}+N_{2}$ and $P=\left(N_{1}-N_{2}\right) /\left(N_{1}+N_{2}\right)$, respectively. The lattice model is adopted here for simplicity, but we have confirmed that the discreteness of the lattice is negligible by assuming the small particle density $N / N_{\mathrm{L}} \leq 0.1$, where $N_{\mathrm{L}}=L \times L$ is the number of lattice sites. Therefore, the following results are valid for continuous systems without lattices in the two-dimensional space. We take the unit of length $d$ so that $1 / 2 m d^{2}=t=1$, where $m$ is the mass of atoms. The last term of eq. (1) describes the $s$-wave attractive interaction. We assume $U / t=-5$ throughout this paper.

The trap potential is assumed to be $V(r)=\frac{1}{2} \omega_{\text {ho }}\left(r / r_{0}\right)^{2}+$ $\omega_{\text {tr }} \exp (-r / \xi)$ with $\xi=5$. This potential describes the harmonic trap for $\omega_{\text {tr }}=0$ and the toroidal trap for $\omega_{\text {tr }} \neq 0$. We found that the A-FFLO state is stabilized for any $\omega_{\text {tr }} / \omega_{\text {ho }}>0$, whose reason will be discussed later. We here show the results for the toroidally trapped system with $\omega_{\text {tr }} / \omega_{\text {ho }}=\frac{2}{3}$ and $\omega_{\text {tr }} / \omega_{\text {ho }}=\frac{1}{4}$ and for the harmonically trapped system with $\omega_{\text {tr }} / \omega_{\text {ho }}=0$.

We analyze the model on the basis of the mean field BdG equation. Details of the calculation have been shown in Ref. 7. It has been shown that the effects of the fluctuation beyond the $\mathrm{BdG}$ equation do not alter the qualitative results [7].

Figure 1 shows the spatial dependence of the superfluid order 
$\omega_{\mathrm{tr}} / \omega_{\mathrm{ho}}=2 / 3$ (a) $\mathrm{P}=0$

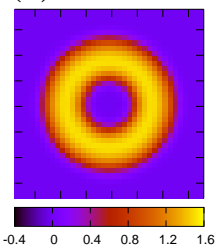

$\omega_{\mathrm{tr}} / \omega_{\mathrm{ho}}=1 / 4$

(d) $\mathrm{P}=0$

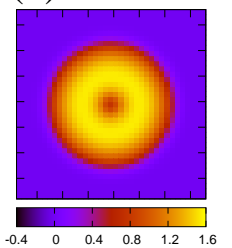

$\omega_{\mathrm{tr}} / \omega_{\mathrm{ho}}=0$

(g) $\mathrm{P}=0$

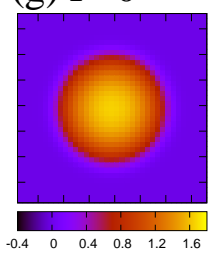

(b) $\mathrm{P}=0.39$

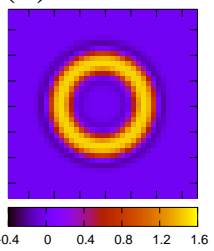

(e) $\mathrm{P}=0.48$

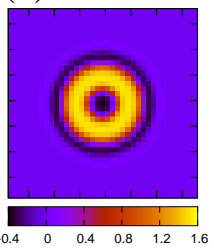

(h) $\mathrm{P}=0.56$

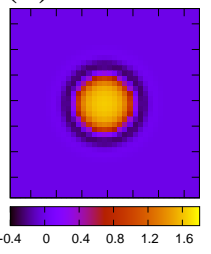

(f) $\mathrm{P}=0.69$

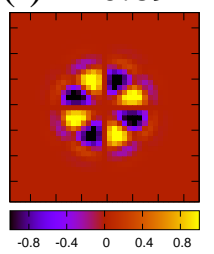

(c) $\mathrm{P}=0.49$

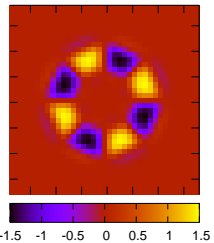

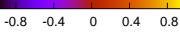
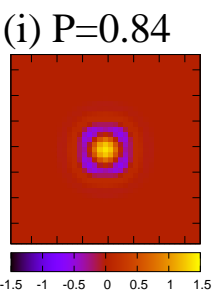

Figure 1: Spatial dependence of the superfluid order parameter $\Delta(\vec{r})=U<$ $c_{\vec{r}, 1} c_{\vec{r}, 2}>$ at $T / t=0.001$, where $T$ is the temperature. We assume $\omega_{\text {tr }} / \omega_{\text {ho }}=$ $2 / 3, \omega_{\text {tr }} / \omega_{\text {ho }}=1 / 4$, and $\omega_{\text {tr }} / \omega_{\text {ho }}=0$, in (a-c), (d-f), and (g-i), respectively. The population imbalance is shown in the figure. (a), (d), and (g) show the BCS state, while (b), (e), (h), and (i) show the R-FFLO state. The rotation symmetry is conserved in these states, but broken in the A-FFLO state in (c) and (f). See the text for details. We assume $N / N_{\mathrm{L}}=0.1$ in (a-f) and $N / N_{\mathrm{L}}=0.07$ in (g-i).

parameter for various trap potential and population imbalance. Figures. 1(a-c) show the results for $\omega_{\text {tr }} / \omega_{\text {ho }}=\frac{2}{3}$. Because the fermion atoms are accumulated in the highly toroidal trap, the superfluid order parameter shows a toroidal shape for $P=0$ (Fig 1(a)). With increasing the imbalance, the order parameter changes the sign along the radial direction as shown in Fig. 1(b), but no spatial symmetry is broken. This is called the radial FFLO (R-FFLO) state. The A-FFLO state is stabilized for $P>$ 0.43 , and its typical spatial structure is shown in Fig. 1(c). It is clearly shown that the rotation symmetry is spontaneously broken in the A-FFLO state. We calculated the particle density and the local population imbalances of the A-FFLO state, and pointed out several intriguing spatial structures which may be observed in future experiments [7].

The A-FFLO state is also realized for a weakly toroidal trap $\omega_{\text {tr }} / \omega_{\text {ho }}=\frac{1}{4}$, as shown in Figs. 1(d-f). We do not see the clear toroidal structure of superfluid order parameter at $P=0$ (Fig. 1(d)), however, the moderate imbalance $P \sim 0.5$ leads to the clear signature of the toroidal trap as shown in Fig. 1(e) where the R-FFLO state is realized. In Fig. 1(e), the sign change along the radial direction induces the quasione-dimensional structure of superfluid order parameter. This quasi-one-dimensional structure is a characteristic feature of the toroidally trapped system as shown in Fig. 1(a). Thus, the feature of the toroidal trap becomes obvious in the imbalanced gases. Because the FFLO state is stable in the quasione-dimensional system [9], the sign change of the order parameter along the angular direction occurs as in Figs. 1(c) and 1(f). Thus, the A-FFLO state is an analog of the quasi-onedimensional FFLO state. Even for the system which is not originally quasi-one-dimensional, the self-one-dimensionalization of the superfluid component occurs due to the formation of the R-FFLO state, and therefore the A-FFLO state is stabilized in the highly imbalanced gases. Since this mechanism is relevant even for a significantly weak toroidal trap $\omega_{\text {tr }} / \omega_{\text {ho }}>0$, the presence of the A-FFLO state is generally expected in the toroidal trap.

Because the self-one-dimensionalization discussed above does not occur in the harmonic trap with $\omega_{\text {tr }} / \omega_{\text {ho }}=0$, the AFFLO state is not stabilized in the harmonic trap. We stress again that this is the reason why the FFLO state has never been observed in the cold fermion gases. Figures. 1(g-i) show that the rotation symmetry is not broken in the harmonic trap. We understand this result by considering the distribution of fermion atoms. The particle density is the largest at the center of the harmonic trap. Therefore, the superfluid phase is stable at the trap core, while the superfluidity is suppressed around the trap edge by increasing the population imbalance. Since the order parameter at the trap core vanishes in the A-FFLO state, the AFFLO state is highly unstable in the harmonic trap. Thus, the harmonic trap does not produce the broken spatial symmetry in the imbalanced superfluid gases.

\section{Summary and Discussion}

We have shown that the A-FFLO state is stabilized in the population imbalanced fermion gases confined in the toroidal trap, but not in the harmonic trap. The formation of the R-FFLO state leads to the self-one-dimensionalization of the superfluid gas and stabilizes the A-FFLO state in the highly imbalanced gases. The search of the FFLO state in cold fermion gases has been fruitless probably because the experiments were carried out for the harmonically trapped gases. It is difficult to detect the FFLO state in the harmonic trap since no space symmetry breaking occurs. On the other hand, the rotation symmetry is spontaneously broken in the A-FFLO state. We suggest that the experiment in the toroidal trap will realize the FFLO state with a broken rotation symmetry and will obtain the unambiguous evidence for the FFLO state which has been searched for more than 40 years after the theoretical predictions [2, 3].

\section{References}

[1] S. Giorgini, L. P. Pitaevskii, and S. Stringari, Rev. Mod. Phys. 80 (2008) 1215.

[2] P. Fulde and R. A. Ferrell, Phys. Rev. 135 (1964) A550.

[3] A. I. Larkin and Yu. N. Ovchinnikov, Zh. Eksp. Teor. Fiz. 47 (1964) 1136 [Sov. Phys. JETP 20 (1965) 762.]

[4] M. W. Zwierlein et al., Science 311 (2006) 492.

[5] G. B. Partridge et al., Science 311 (2006) 503. 
[6] T. Mizushima, M. Ichioka, and K. Machida, J. Phys. Soc. Jpn. 76 (2007) 104006.

[7] Y. Yanase, Phys. Rev. B 80 (2009) 220510(R).

[8] For a work on the FFLO state with rotation symmetry, H.Shimahara, Czech. J. Phys. 46 (1996) Suppl.S2, 561.

[9] Y. Matsuda and H. Shimahara, J. Phys. Soc. Jpn. 76 (2007) 051005 and references there in. 